\title{
Influence of Coronary Risk Factors on Coronary Events in Japanese High-Risk Hypertensive Patients - Primary and Secondary Prevention of Ischemic Heart Disease in a Subanalysis of the Candesartan Antihypertensive Survival Evaluation in Japan (CASE-J) Trial -
}

Kenji Ueshima, MD, PhD; Koji Oba, BSc; Shinji Yasuno, MD, PhD; Akira Fujimoto, BSc; Shiro Tanaka, PhD; Toshio Ogihara, MD, PhD; Takao Saruta, MD, PhD; Kazuwa Nakao, MD, PhD

\begin{abstract}
Background: The Candesartan Antihypertensive Survival Evaluation in Japan (CASE-J) trial was conducted to compare the effects of candesartan and amlodipine on cardiovascular events in Japanese high-risk hypertensive patients. The aim of the present subanalysis was to evaluate the influence of coronary risk factors on coronary events in these patients as an observational study irrespective of allocated drugs.
\end{abstract}

Methods and Results: The adjusted hazard ratios (HRs) of the association of baseline risk factors including gender, age, allocated drugs, body mass index, systolic/diastolic blood pressure (SBP/DBP), diabetes mellitus (DM), hyperlipidemia $(\mathrm{HL})$, smoking, left ventricular hypertrophy, previous ischemic heart disease (IHD), previous cerebrovascular events, and chronic kidney disease (CKD) with coronary events in 4,703 patients who were enrolled in the CASE-J trial, were examined. The coronary events occurred in 83 patients, and were significantly associated with previous IHD, DM, male sex, CKD, and low DBP. Significant predictors were previous IHD (HR, 3.89), DM (HR, 3.10), male sex (HR, 1.81), CKD (HR, 1.60), and low DBP (HR, 1.36), respectively. In 4,107 patients without previous IHD, DM (HR, 4.88), HL (HR, 2.67), and DBP (HR, 1.39) were significantly associated with the risk of coronary events, while male sex (HR, 3.03), CKD (HR, 2.44), and DM (HR, 2.15) were in 596 patients with previous IHD.

Conclusions: DM is the important factor in both primary and secondary prevention of coronary events. Comprehensive risk management including surveillance of DM, CKD and $\mathrm{HL}$ is needed for preventing coronary events, in addition to blood pressure control. (Circ $J$ 2011; 75: 2411-2416)

Key Words: Coronary event; Coronary risk factor; Hypertension; Japanese; Prevention

A lthough the current incidence of acute myocardial infarction in Japan is still lower than that in North America and Europe, ${ }^{1}$ a recent report indicated that there has been a steady trend of increasing incidence of acute myocardial infarction during the past 30 years in the Japanese population. $^{2}$ We should be deeply concerned about this increase of coronary events in Japan with regard to westernized lifestyle and aging of the population. Hypertension has been one of the major risk factors for cardiovascular (CV) events, and $\mathrm{CV}$ risks are well known to cluster in hypertensive patients. ${ }^{3,4}$ It is important to consider coronary risk factors in hypertensive patients when we try to reduce the incidence of ischemic heart disease (IHD) in terms of primary and secondary prevention.

\section{Editorial p 2316}

The Candesartan Antihypertensive Survival Evaluation in Japan (CASE-J) trial was conducted to compare the effects of the angiotensin II receptor blocker candesartan and the calcium channel blocker amlodipine on the incidence of $\mathrm{CV}$ events in Japanese high-risk hypertensive patients.,6 The CASE-J trial found that candesartan and amlodipine equally suppressed total CV mortality and morbidity in high-risk hy-

Received November 18, 2010; revised manuscript received April 25, 2011; accepted May 18, 2011; released online July 20, 2011 Time for primary review: 54 days

EBM Research Center (K.U., K.O., S.Y., A.F., K.N.), Department of Medicine and Clinical Science (K.N.), Kyoto University Graduate School of Medicine, Kyoto; Translational Research Center, Kyoto University Hospital, Kyoto (S.T.); Osaka General Medical Center, Osaka (T.O.); and Keio University, Tokyo (T.S.), Japan

Mailing address: Kenji Ueshima, MD, PhD, EBM Research Center, Kyoto University Graduate School of Medicine, Yoshida-Konoechou, Sakyo-ku, Kyoto 606-8501, Japan. E-mail: kenji.ueshima@at3.ecs.kyoto-u.ac.jp

ISSN-1346-9843 doi:10.1253/circj.CJ-10-1161

All rights are reserved to the Japanese Circulation Society. For permissions, please e-mail: cj@j-circ.or.jp 
Table 1. Selected Coronary Risk Factors and Definitions for Baseline Characteristics

Gender

Age

Allocated drugs: candesartan or amlodipine

$\mathrm{BMI}$ at baseline

SBP at baseline

DBP at baseline

Smoking (including previous history of smoking)

DM: at least one of the following factors: fasting blood glucose $\geq 126 \mathrm{mg} / \mathrm{dl}$, causal blood glucose $\geq 200 \mathrm{mg} / \mathrm{dl}$,

$\mathrm{HbA}_{1 \mathrm{c}} \geq 6.5 \%$, 2-h blood glucose on $75-\mathrm{g}$ OGTT $\geq 200 \mathrm{mg} / \mathrm{dl}$, or current treatment with hypoglycemic agent

$\mathrm{HL}$ : current treatment with anti-lipidemic agent

Cerebrovascular disease: history of cerebral hemorrhage, cerebral infarction, or transient ischemic attack until 6 months prior to the screening

LVH: thickness of the posterior wall of left ventricle or thickness of the wall of interventricular septum $\geq 12 \mathrm{~mm}$ on echocardiography or Sv1 + Rv5 $\geq 35 \mathrm{~mm}$ on electrocardiography

IHD: angina pectoris, and a past history ( $\geq 6$ months before giving informed consent) of myocardial infarction

CKD: proteinuria $\geq+1$ or renal impairment (estimated glomerular filtration rate $<60 \mathrm{ml} \cdot \mathrm{min}^{-1} \cdot 1.73 \mathrm{~m}^{-2}$ by a predictive equation) within 3 months at the time of giving informed consent

Anti-hypertensive medication prior to screening

BMI, body mass index; SBP, systolic blood pressure; DBP, diastolic blood pressure; DM, diabetes mellitus; OGTT, oral glucose tolerance test; $\mathrm{HL}$, hyperlipidemia; $\mathrm{LVH}$, left ventricular hypertrophy; IHD, ischemic heart disease; CKD, chronic kidney disease.

\begin{tabular}{lccc|}
\hline Table 2. Baseline Subject Characteristics & & & \\
& $\begin{array}{c}\text { All patients } \\
(\mathbf{n = 4 , 7 0 3 )}\end{array}$ & $\begin{array}{c}\text { Previous IHD (+) } \\
(\mathbf{n}=\mathbf{5 9 6 )}\end{array}$ & $\begin{array}{c}\text { Previous IHD (-) } \\
(\mathbf{n}=\mathbf{4 , 1 0 7 )}\end{array}$ \\
Gender, male (\%) & $2,597(55.2)$ & $397(66.6)$ & $2,200(53.6)$ \\
Age (years), mean \pm SD & $63.9 \pm 10.5$ & $67.2 \pm 8.7$ & $63.4 \pm 10.7$ \\
Allocated drugs, candesartan (\%) & $2,354(50.1)$ & $298(50.0)$ & $2,056(50.1)$ \\
BMl, mean \pm SD & $24.6 \pm 3.7$ & $24.3 \pm 3.3$ & $24.6 \pm 3.7$ \\
SBP, mean \pm SD & $162.9 \pm 14.2$ & $157.5 \pm 12.3$ & $163.6 \pm 14.3$ \\
DBP, mean \pm SD & $91.7 \pm 11.2$ & $86.9 \pm 9.1$ & $92.4 \pm 11.3$ \\
Smoking (no) (\%) & $3,205(68.1)$ & $346(58.1)$ & $2,859(69.5)$ \\
DM (yes) (\%) & $2,018(42.9)$ & $203(34.1)$ & $1,815(44.2)$ \\
HL (yes) (\%) & $2,078(67.3)$ & $325(54.5)$ & $1,753(42.7)$ \\
LVH (yes) (\%) & $1,612(34.3)$ & $178(29.9)$ & $1,434(34.9)$ \\
Previous IHD (yes) (\%) & $596(12.7)$ & $596(100)$ & $0(0)$ \\
Previous stroke (yes) (\%) & $473(10.1)$ & $41(6.9)$ & $432(10.5)$ \\
CKD (yes) (\%) & $2,571(54.7)$ & $296(49.7)$ & $2,275(55.4)$ \\
Previous AHD (yes) (\%) & $3,165(67.3)$ & $512(85.9)$ & $2,653(64.6)$ \\
\hline
\end{tabular}

AHD, anti-hypertensive drug. Other abbreviations see in Table 1.

pertensive patients under strict blood pressure (BP) control.

Although some Japanese cohort studies have been performed to clarify the coronary risk factors, this trial was an observational study irrespective of allocated drugs, and the purpose of the present subanalysis was to clarify the adverse effects of coronary risks in Japanese high-risk hypertensive patients. Although prior cohort studies focused on the general population, the present study focused on those subjects taking anti-hypertensive drugs. Therefore, the influence of risk factors on coronary events under control could be clarified.

\section{Methods}

\section{Study Design}

The CASE-J trial was a prospective, multicenter, randomized, open-label, active-controlled, 2-arm parallel-group comparison study to evaluate the efficacy of candesartan and amlodipine for reducing the incidence of $\mathrm{CV}$ events in high-risk hypertensive patients. ${ }^{5,6}$ The rationale and complete design of the CASE-J trial have been previously reported. ${ }^{5}$ Briefly, 4,728 patients with high-risk hypertension were randomly assigned to either a candesartan- or amlodipine-based treatment regimen. High risk was defined as the presence of any one of the following: (1) severe hypertension (systolic BP/ diastolic BP [SBP/DBP] $\geq 180 / 110 \mathrm{mmHg}$; (2) type 2 diabetes mellitus (DM); (3) history of stroke or transient ischemic attack > 6 months prior to screening; (4) left ventricular hypertrophy (LVH; SV1+RV5 $\geq 3.5 \mathrm{mV}$ on electrocardiography [ECG] and/or LV wall thickness $\geq 12 \mathrm{~mm}$ on echocardiography), angina pectoris, or a history of myocardial infarction $>6$ months prior to screening; (5) proteinuria or serum creatinine concentration $\geq 1.3 \mathrm{mg} / \mathrm{dl}$; and (6) arteriosclerotic peripheral artery obstruction. ${ }^{5}$ The target BP was determined according to the guideline proposed by the Japanese Society of Hypertension. ${ }^{7}$ Finally, 4,703 randomly assigned patients were included in the analysis. 


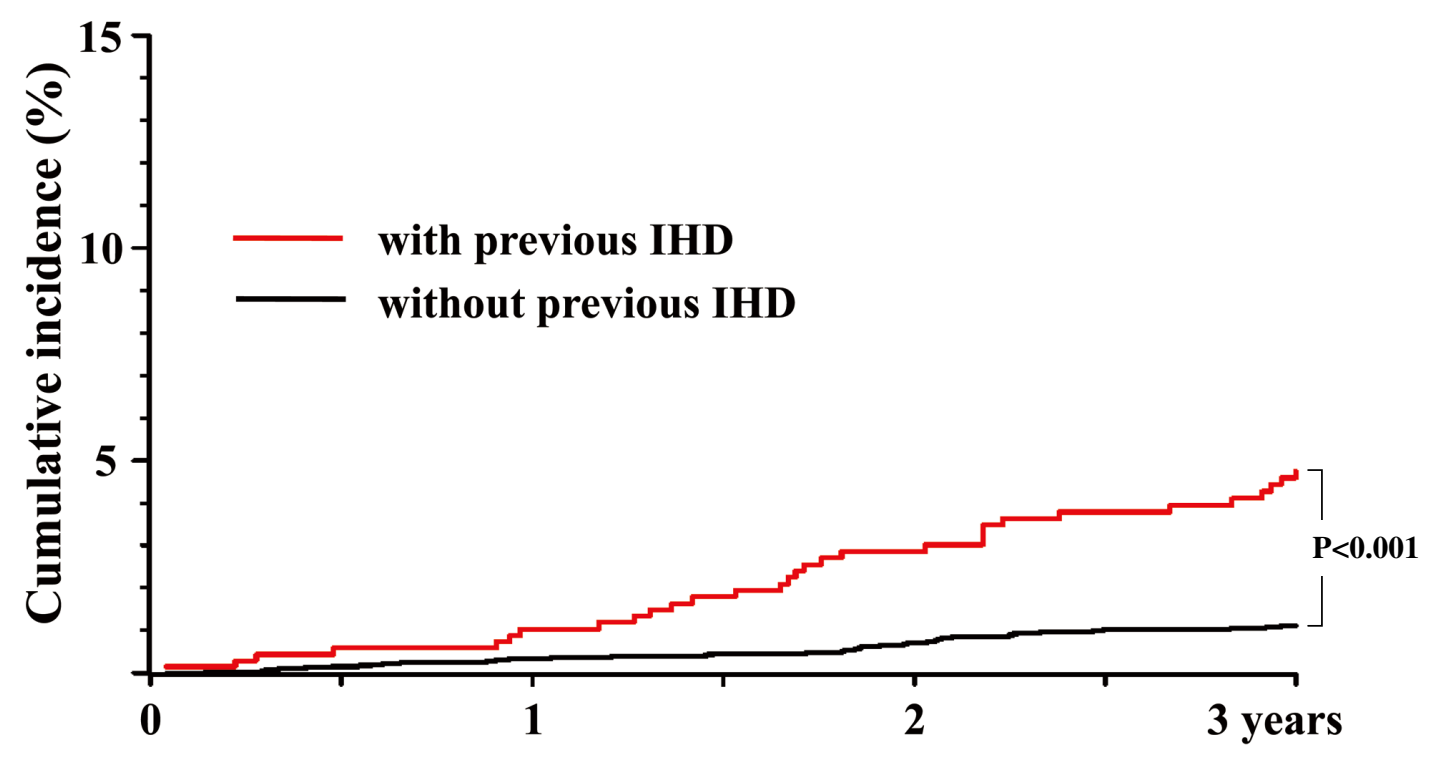

Figure. Cumulative incidence of the primary composite endpoint adjusted for baseline characteristics in patients without ischemic heart disease (IHD) at baseline and with IHD at baseline.

\section{Outcome Measurements}

In the CASE-J trial, the primary endpoint was the first fatal/ non-fatal CV event (a composite of cardiac events including sudden death, angina pectoris, acute myocardial infarction, or heart failure; cerebrovascular events including stroke or transient ischemic attack; renal events including serum creatinine concentration $\geq 4.0 \mathrm{mg} / \mathrm{dl}$, doubling of the serum creatinine concentration, or end-stage renal disease; and vascular events including dissecting aortic aneurysm or arteriosclerotic occlusion of a peripheral artery). ${ }^{6}$ In the present subanalysis, we focused on the incidence of coronary events. Thus, the endpoint of this subanalysis was coronary events, and they were defined as a composite of sudden death (unexpected death happening within $24 \mathrm{~h}$ without external causes), angina pectoris (nitrate-sensitive chest pain and ischemic ECG changes during chest pain or stress testing), and acute myocardial infarction (nitrate-resistant chest pain, elevated myocardial specific enzymes, and typical ECG signs). Event evaluation was performed independently by the Event Evaluation Committee, which was blinded to the assigned treatment groups and adjudicated according to the prespecified protocol criteria as described here. ${ }^{6}$

\section{Baseline Characteristics}

Background coronary risk factors such as gender, age, allocated drugs, body mass index (BMI), SBP, DBP, smoking, type $2 \mathrm{DM}$, hyperlipidemia (HL), LVH, history of previous IHD, history of cerebrovascular disease, chronic kidney disease (CKD), and anti-hypertensive medication prior to the screening were analyzed (Table 1). Baseline characteristics of enrolled patients are listed in Table 2. Moreover, we focused on the presence or absence of previous IHD including angina pectoris, and a past history ( $\geq 6$ months before giving informed consent) of myocardial infarction, which were 1 of the inclusion criteria in the CASE-J trial. Enrolled patients were divided into 4,107 patients without previous IHD and 596 patients with previous IHD. In the present study, risk factors for the first coronary event were assessed in patients without previous IHD and those for the recurrence of coronary events were done in patients with previous IHD. Their baseline characteristics are also given in Table 2 . When we analyzed the data of patients with or without coronary risk factors as an observational study irrespective of allocated drugs, there were statistical differences between these 2 groups. Analyses were then adjusted by baseline characteristics as described in the following section.

\section{Statistical Analysis}

Data are expressed as mean \pm SD or proportions. Risk-adjusted cumulative incidence of coronary events was calculated using the corrected group prognosis method, ${ }^{8}$ with adjustment for baseline characteristics, including history of prior anti-hypertensive treatment, allocated drugs, age, sex, BMI, type 2 DM, history of cerebrovascular disease, history of IHD, renal dysfunction, history of vascular disease, SBP and DBP at baseline. The hazard ratio (HR) and 95\% confidence intervals (95\%CIs) were estimated using Cox regression analysis. We also used multiple Cox regression analysis to examine the association between rate of coronary events and coronary risk factors (gender, age, allocated drugs, BMI, SBP, DBP, smoking, DM, HL, LVH, previous IHD, previous cerebrovascular disease, $\mathrm{CKD}$, and previous anti-hypertensive medication). All statistical tests were 2-sided with an alpha level of 0.05, and were performed using SAS version 9.1 (SAS Institute, Cary, NC, USA).

\section{Results}

\section{Changes in BP}

Blood pressure was generally controlled to $<140 / 80 \mathrm{mmHg}$ in both groups. Mean SBP/DBP was 162.9 $\pm 14.2 / 91.7 \pm$ $11.2 \mathrm{mmHg}$ at baseline and $135.4 \pm 11.9 / 77.0 \pm 9.2 \mathrm{mmHg}$ after 3 years. While the mean SBP/DBP in patients without previous IHD was $163.6 \pm 14.3 / 92.4 \pm 11.3 \mathrm{mmHg}$ at baseline and 
Table 3. Prognostic Value of Coronary Risk Factors in All Enrolled Patients

\begin{tabular}{|c|c|c|}
\hline & HR $(95 \% \mathrm{Cl})$ & $P$ value \\
\hline Male (vs. female)* & $1.81(1.06-3.10)$ & 0.031 \\
\hline Age: 10-year increase & $1.09(0.83-1.44)$ & 0.525 \\
\hline Amlodipine (vs. candesartan) & $1.32(0.85-2.04)$ & 0.214 \\
\hline BMI: $1-\mathrm{kg} / \mathrm{m}^{2}$ increase & $1.01(0.83-1.44)$ & 0.525 \\
\hline SBP (at entry): $10-\mathrm{mmHg}$ increase & $1.14(0.96-1.37)$ & 0.145 \\
\hline DBP (at entry): $10-\mathrm{mmHg}$ decrease* & $1.36(1.09-1.68)$ & 0.006 \\
\hline Smoking: no (vs. yes) & $1.02(0.62-1.69)$ & 0.945 \\
\hline DM: no (vs. yes)* & $3.10(1.90-5.04)$ & $<0.001$ \\
\hline HL: no (vs. yes) & $1.36(0.86-2.14)$ & 0.186 \\
\hline LVH: no (vs. yes) & $1.26(0.80-1.99)$ & 0.319 \\
\hline Previous IHD: no (vs. yes) ${ }^{*}$ & $3.89(2.40-6.31)$ & $<0.001$ \\
\hline Previous stroke: no (vs. yes) & $0.78(0.34-1.82)$ & 0.569 \\
\hline CKD: no (vs. yes) ${ }^{\star}$ & $1.60(1.01-2.54)$ & 0.046 \\
\hline Previous AHD: no (vs. yes) & $1.54(0.85-2.81)$ & 0.159 \\
\hline
\end{tabular}

$\mathrm{HR}$, hazard ratio; $\mathrm{Cl}$, confidence interval. Other abbreviations see in Tables 1,2 .

${ }^{*} \mathrm{P}<0.05$.

\begin{tabular}{|lcr|}
\hline $\begin{array}{c}\text { Table 4. Prognostic Value of Coronary Risk Factors in Patients } \\
\text { Without Previous IHD (Primary Prevention) }\end{array}$ \\
& HR (95\%Cl) & P value \\
& $1.44(0.73-2.84)$ & 0.294 \\
Male (vs. female) & $1.18(0.83-1.64)$ & 0.364 \\
Age: 10 -year increase & $1.07(0.61-1.87)$ & 0.811 \\
Amlodipine (vs. candesartan) & $0.99(0.91-1.07)$ & 0.810 \\
BMI: $1-k g / m^{2}$ increase & $1.18(0.94-1.54)$ & 0.160 \\
SBP (at entry): 10-mmHg increase & $1.39(1.05-2.18)$ & 0.020 \\
DBP (at entry): 10-mmHg decrease & $0.70(0.35-1.37)$ & 0.296 \\
Smoking: no (vs. yes) & $4.88(2.35-10.16)$ & $<0.001$ \\
DM: no (vs. yes) & $2.67(1.47-4.85)$ & 0.001 \\
HL: no (vs. yes) & $1.21(0.65-2.24)$ & 0.555 \\
LVH: no (vs. yes) & $1.03(0.40-2.63)$ & 0.957 \\
Previous stroke: no (vs. yes) & $1.21(0.67-2.19)$ & 0.521 \\
CKD: no (vs. yes) & $1.19(0.62-2.28)$ & 0.595 \\
Previous AHD: no (vs. yes) & &
\end{tabular}

Abbreviations see in Tables 1-3.

${ }^{*} \mathrm{P}<0.05$.

Table 5. Prognostic Value of Coronary Risk Factors in Patients With Previous IHD (Secondary Prevention)

\begin{tabular}{lcc} 
& HR (95\%Cl) & P value \\
Male (vs. female) & (9.05 (1.15-14.63) & 0.025 \\
Age: 10 -year increase & $1.00(0.63-1.28)$ & 0.992 \\
Amlodipine (vs. candesartan) & $1.77(0.87-3.63)$ & 0.117 \\
BMl: 1-kg/m² increase & $1.03(0.91-1.15)$ & 0.673 \\
SBP (at entry): 10-mmHg increase & $1.16(0.84-1.57)$ & 0.360 \\
DBP (at entry): 10-mmHg decrease & $1.22(0.82-1.81)$ & 0.323 \\
Smoking: no (vs. yes) & $1.54(0.72-3.45)$ & 0.268 \\
DM: no (vs. yes) & $2.15(1.06-4.38)$ & 0.035 \\
HL: no (vs. yes) & $0.54(0.26-1.12)$ & 0.098 \\
LVH: no (vs. yes) & $1.39(0.69-2.81)$ & 0.360 \\
Previous stroke: no (vs. yes) & $0.34(0.05-2.54)$ & 0.290 \\
CKD: no (vs. yes) & $2.44(1.16-5.13)$ & 0.018 \\
Previous AHD: no (vs. yes) & $5.13(0.68-38.49)$ & 0.112 \\
\hline
\end{tabular}

Abbreviations see in Tables 1-3.

${ }^{*} \mathrm{P}<0.05$
$135.7 \pm 12.0 / 77.3 \pm 9.3 \mathrm{mmHg}$ after 3 years, the mean SBP/DBP in patients with previous IHD was $157.5 \pm 12.3 / 86.9 \pm 9.1 \mathrm{mmHg}$ at baseline and $133.2 \pm 11.4 / 75.1 \pm 8.2 \mathrm{mmHg}$ after 3 years. But both SBP and DBP in patients with previous IHD were slightly but significantly lower than in those without previous IHD at baseline and after 3 years $(\mathrm{P}<0.001$, respectively).

\section{Prognostic Value of Coronary Risk Factors for Coronary Event Rate}

During 3.2 \pm 0.9 years of follow-up (5th-95th percentile interval, 1.0-4.2), coronary events occurred in 50 patients without previous IHD $(1.2 \%$; 15 sudden death, 11 angina pectoris, 24 myocardial infarction) at baseline for a rate of 3.7 per 1000 patient-years and in 33 patients with previous IHD $(5.5 \%$; 11 sudden death, 11 angina pectoris, 11 myocardial infarction) at baseline for a rate of 16.9 per 1,000 patient-years (adjusted HR, 3.89; 95\%CI: 2.40-6.31; P<0.001; Figure). We also evaluated the prognostic value of the coronary risk factors in all enrolled patients. As shown in Table 3, the onset of coronary events was significantly associated with previous IHD (adjusted HR, 3.89), DM (adjusted HR, 3.10; 95\%CI: 1.905.04; $\mathrm{P}<0.001$ ), male sex (adjusted HR, 1.81; 95\%CI: 1.063.10; $\mathrm{P}=0.031$ ), CKD (adjusted HR, 1.60; 95\%CI: 1.01-2.54; $\mathrm{P}=0.046)$, and DBP (10-mmHg decrease, adjusted HR, 1.36; 95\%CI: $1.09-1.68 ; \mathrm{P}=0.006)$.

In addition, the prognostic value of the coronary risk factors for each event category was evaluated in patients without previous IHD (primary prevention) and in patients with previous IHD (secondary prevention), respectively. As shown in Table 4, the onset of coronary events in patients without previous IHD was significantly associated with DM (adjusted HR, 4.88; 95\%CI: 2.35-10.16; P<0.001), HL (adjusted HR, 2.67; 95\%CI: $1.47-4.85 ; \mathrm{P}=0.001)$, and DBP $(10 \mathrm{mmHg}$ decrease, adjusted HR, 1.39; 95\%CI: 1.05-2.18; $\mathrm{P}=0.02)$. In patients with previous IHD, the incidence of coronary events was significantly associated with male sex (adjusted HR, 3.05; 95\% CI: 1.15-14.63; $\mathrm{P}=0.025$ ), CKD (adjusted HR, 2.44; 95\% CI: 1.16-5.13; $\mathrm{P}=0.018$ ), and DM (adjusted HR, 2.15; 95\%CI: 1.06-4.38; $\mathrm{P}=0.035$; Table 5). Although a significant difference was observed in the risk factors between primary prevention and secondary prevention, interactions between the coronary risk factors and previous IHD were not significant except for HL. Notably, DM was the common predictor of coronary events both in primary and secondary prevention.

\section{Discussion}

Increases in BP loads induce myocardial remodeling, such as cardiac hypertrophy, myocardial fibrosis and coronary endothelial damage. Progression of myocardial remodeling and coronary atherosclerosis leads to coronary artery disease, heart failure, arrhythmia and sudden death. In men, morbidity and mortality rates due to coronary artery disease increase by approximately $15 \%$ with a $10-\mathrm{mmHg}$ increase in SBP. ${ }^{9}$ Conventional anti-hypertensive drug therapy primarily using diuretics and $\beta$-blockers, however, does not markedly reduce the incidence of coronary artery disease, whereas it markedly decreases the incidence of stroke. ${ }^{10}$ Coronary risk factors other than hypertension may have a markedly different impact on the occurrence of coronary artery disease. ${ }^{11}$ Accordingly, the aim of the present study was to clarify the influence of coronary risk factors on coronary events in Japanese high-risk hypertensive patients as a subanalysis of the CASE-J trial. The follow-up rate and coronary event evaluation are markedly reliable from this cohort study. Furthermore, although some 
Japanese cohort studies were performed to clarify coronary risk factors, ${ }^{12-16}$ this subanalysis focused on the subjects under anti-hypertensive treatment. Therefore, the adverse effects of coronary risk factors under BP control could be evaluated.

First, we demonstrated that a history of previous IHD was the strongest predictor for coronary events in all enrolled patients. Because the prognosis of patients with previous IHD is worse, a sufficient reduction of BP in these patients is important to decrease CV events and mortality. ${ }^{6,17,18}$ In the Japan Lipid Intervention Trial, the incidence of coronary events in patients with a history of IHD was 5-fold higher than in patients without this. ${ }^{19}$ In addition, Iso reported that the percentage of preventable IHD was $34 \%$ in men and $17 \%$ in women for control of hypertension. ${ }^{8}$ A total of $80-90 \%$ of hypertensive patients in their $30 \mathrm{~s}$ and $40 \mathrm{~s}$, however, are untreated in Japan. ${ }^{9}$ For reduction of coronary events, population-wide health education is needed for prevention of high BP through lifestyle modifications.

With regard to primary prevention, we also found that DM, HL, and DBP decrease at baseline were significant predictors of occurrence of $\mathrm{CV}$ events. DM is well known to be 1 of the most important causes of CV events. Berman et al identified a significant impact of DM on cardiac risk and found that diabetic women have a significantly greater risk of cardiac death than other patients. ${ }^{20}$ Haffner et al found that diabetic patients without previous myocardial infarction in a Finnish population-based study had as high a risk of myocardial infarction as non-diabetic patients with previous myocardial infarction. ${ }^{21}$ In Japanese people, Nishimura et a; also demonstrated that the $\mathrm{CV}$ event rates were similar among nondiabetic patients with prior myocardial infarction and diabetic patients without prior myocardial infarction. ${ }^{22}$ It is also well established that HL is a major cause of IHD. According to a Japanese autopsy study, the myocardial infarction size among urban men with HL was large, as also observed in Western populations. ${ }^{23}$ Mabuchi et al found that patients with a total cholesterol concentration $\geq 240 \mathrm{mg} / \mathrm{dl}$ developed coronary heart disease more often than patients with total cholesterol $<240 \mathrm{mg} / \mathrm{dl} .{ }^{19}$ The serum low-density lipoprotein cholesterol concentration positively correlated with incidence of the coronary events. The percentage of preventable coronary heart disease was $5 \%$ in men and $9 \%$ in women for control of DM, and $5 \%$ in men and $8 \%$ in women for control of HL. ${ }^{8}$ In the present study, the lower DBP was extracted from the database as a significant predictor of coronary events. Several observational studies have provided evidence suggesting that pulse pressure was a better predictor of $\mathrm{CV}$ events than mean pressure. ${ }^{24-26}$ In the Multiple Risk Factor Intervention Trial, the greatest risk was observed in subjects with the highest SBP $(\geq 160 \mathrm{mmHg})$ and lowest DBP $(<70 \mathrm{mmHg})$ at enrollment. Furthermore, the 2-year probability of a major CV endpoint increased with lower DBP at any given level of SBP. ${ }^{26}$ Because patients at high risk of coronary events had increased arterial stiffness, lower DBP was identified as a significant marker of coronary events. We also reported that a strong association with risk of new-onset DM was seen in patients with higher pulse pressures, arising mainly due to the lower DBP. ${ }^{27}$

With regard to secondary prevention, we demonstrated that male sex, CKD, and DM were significant predictors for coronary events. Hypertension causes functional or structural changes to varying degrees in the kidney from an early stage, and renal dysfunction may also cause hypertension. Renal dysfunction and proteinuria are known to be risk factors for end-stage renal failure, ${ }^{28,29}$ but they have also recently been found to be strong risk factors for CV disease..$^{16,30,31}$ The con- cept of CKD was introduced for preventing the occurrence of $\mathrm{CV}$ disease as well as renal insufficiency by the early detection and treatment of renal diseases. ${ }^{32}$ Therefore, strict management of BP as well as treatment of the underlying renal disease is important.

Prevention and treatment for glucose normalization is important in not only primary prevention for coronary events but also in secondary prevention. Elevated blood glucose levels on admission are associated with increased mortality in patients with acute myocardial infarction. In the J-ACCESS study, patients with suspected or confirmed IHDs were classified into 4 groups according to DM and prior myocardial infarction. The prognosis was worst among diabetic patients with prior myocardial infarction. ${ }^{22}$ Because the risks of $\mathrm{CV}$ events in patients with DM, who achieved SBP/DBP $<130 / 75-$ $79 \mathrm{mmHg}$, were still significantly higher than in those without $\mathrm{DM}$, strict $\mathrm{BP}$ control is needed to reduce CV events in hypertensive patients with DM. ${ }^{33}$ In the present subanalysis, previous IHD including angina pectoris and myocardial infarction was one of the inclusion criteria in the CASE-J trial, and there were several differences in baseline characteristics between previous IHD (-) and previous IHD (+), as shown in Table 2. Therefore, when we evaluated the data of patients with baseline coronary risk factors, the analyses had to be adjusted by the baseline characteristics because of their statistical differences. Interestingly, DM was the common predictor of coronary events both in primary and secondary prevention.

Several limitations in the present study should be noted. This was a post-hoc analysis. The number of coronary events may not be sufficient to analyze the influence of the coronary risk factors on these events. Furthermore, a 3.2-year mean follow-up period may not be sufficient to evaluate the relationship between underlying risks and the incidence of coronary events. The CASE-J trial was extended for 3 years from 2006, it was named the CASE-J Ex and it was an observational study. ${ }^{34}$ The CASE-J Ex may resolve this issue. Moreover, a difference in the significant risk factors between primary and secondary prevention was observed. Moreover, because DM at baseline was diagnosed by each participant doctor, we did not evaluate specific blood sugar or HbA1c. Thus, information on the relationship between blood sugar or $\mathrm{HbA}_{1 \mathrm{c}}$ and the risk of coronary events is not available. Finally, because HL was diagnosed only on taking medications for HL, there is a possibility that the influence of HL on coronary events was underestimated.

In conclusion, previous IHD, DM, male sex, CKD, and DBP decrease are significant risk factors in all enrolled patients to the CASE-J trial. Because DM, HL and DBP decrease are risk factors in patients without previous IHD, lowering blood sugar and cholesterol are still important measures in terms of primary prevention. In addition, because male sex, CKD and DM are risk factors in patients with previous IHD, $\mathrm{CKD}$ and $\mathrm{DM}$ are important issues in terms of secondary prevention. Although there are some differences in the coronary risk factors between primary and secondary prevention in Japanese high-risk hypertensive patients, DM is the important factor in both primary and secondary prevention. Furthermore, comprehensive risk management including surveillance of DM, CKD and HL is needed for preventing coronary events, in addition to BP control. General and particular care of lifestyle-related disease leads to prevention of IHD.

\section{Source of Funding}

The CASE-J trial was funded by EBM Research Center, Kyoto University of Graduate School of Medicine, with an unrestricted grant from Takeda 
Pharmaceutical. The Japanese Society of Hypertension supported the trial.

\section{Disclosures}

Ueshima K, Ogihara T, Saruta T, and Nakao K received honoraria for lectures from Takeda Pharmaceutical and Pfizer Japan. The other authors declare that they have no conflicts of interest.

\section{References}

1. Sekikawa A, Ueshima H, Kadowaki T, El-Saed A, Okamura T, Takamiya $\mathrm{T}$, et al. Less subclinical atherosclerosis in Japanese men in Japan than in White men in the United States in the post-World War II birth cohort. Am J Epidemiol 2007; 165: 617-624.

2. Takii T, Yasuda S, Takahashi J, Ito K, Shiba N, Shirato K, et al. Trends in acute myocardial infarction incidence and mortality over 30 years in Japan: Report from the MIYAGI-AMI Registry Study. Circ J 2010; 74: 93-100.

3. Nakamura Y, Saitoh S, Takagi S, Ohnishi H, Chiba Y, Kato N, et al. Impact of abnormal glucose tolerance, hypertension, and other risk factors on coronary heart disease. Circ $J$ 2007; 71: 20-25.

4. Kohro T, Hayashi D, Okada Y, Yamazaki T, Nagai R; JCAD Investigators. Demographics and changes in medical/interventional treatment of coronary artery disease patients over a 3.5 -year period in Japan: The Japanese Coronary Artery Disease Study: Trend examination. Circ J 2008; 72: 1397-1402.

5. Fukui T, Rahman M, Hayashi K, Takeda K, Higaki J, Sato T, et al. Candesartan Antihypertensive Survival Evaluation in JAPAN (CASE-J) trial of cardiovascular events in high-risk hypertensive patients: Rationale, design, and methods. Hypertens Res 2003; 26: 979-990.

6. Ogihara T, Nakao K, Fukui T, Fukiyama K, Ueshima K, Oba K, et al; Candesartan Antihypertensive Survival Evaluation in Japan trial Group. Effects of candesartan compared with amlodipine in hypertensive patients with high cardiovascular risks: Candesartan antihypertensive survival evaluation in Japan trial. Hypertension 2008; 51: 393-398.

7. Japanese Society of Hypertension Guidelines Subcommittee for the Management of Hypertension. Guidelines for the management of hypertension for general practitioners. Hypertens Res 2001; 24: 613-634.

8. Iso H. Changes in coronary heart disease risk among Japanese. Circulation 2008; 118: 2725-2729.

9. The Japanese Society of Hypertension Guidelines for the Management of Hypertension (JSH 2009). Epidemiology of hypertension. Hypertens Res 2009; 32: 6-10.

10. Moser M, Hebert P, Hennekens CH. An overview of the metaanalyses of the hypertension treatment trials. Arch Intern Med 1991; 151: $1277-1279$.

11. Ueshima K, Yasuno S, Oba K, Fujimoto A, Ogihara T, Saruta T, et al. The effects of cardiac complications on cardiovascular events in Japanese high-risk hypertensive patients: A subanalysis of CASEJ trial. Circ J 2009; 73: 1080-1085.

12. Katsuki S. Epidemiological and clinicopathological study on cerebrovascular disease in Japan. Prog Brain Res 1966; 21: 64-89.

13. Ueda K, Fujii I, Kawano H, Hasuo Y, Yanai T, Kiyohara Y, et al. Severe disability related to cerebral stroke: Incidence and risk factors observed in a Japanese community, Hisayama. J Am Geriatr Soc 1987; 35: 616-622.

14. Obara F, Saitoh S, Takagi S, Shimamoto K. Influence of hypertension on the incidence of cardiovascular disease in two rural communities in Japan: The Tanno-Sobetsu study. Hypertens Res 2007; 30: $677-682$.

15. Ueshima H, Choudhury SR, Okayama A, Hayakawa T, Kita Y, Kadowaki T, et al. Cigarette smoking as a risk factor for stroke death in Japan: NIPPON DATA80. Stroke 2004; 35: 1836-1841.

16. Nakamura K, Okamura T, Hayakawa T, Kadowaki T, Kita Y, Ohnishi H, et al; The NIPPON DATA90 Research Group. Chronic kidney disease is a risk factor for cardiovascular death in a community-based population in Japan: NIPPON DATA90. Circ J 2006; 70: $954-959$.

17. Staessen JA, Wang JG, Thijs L. Cardiovascular protection and blood pressure reduction: A meta-analysis. Lancet 2001; 358: 1305-1315.

18. ALLHAT Officers and Coordinators for the ALLHAT Collaborative Research Group. The Antihypertensive and Lipid-Lowering Treatment to Prevent Heart Attack Trial. Major outcomes in high-risk hypertensive patients randomized to angiotensin-converting enzyme inhibitor or calcium channel blocker vs diuretic: The Antihypertensive and Lipid-Lowering Treatment to Prevent Heart Attack Trial (ALLHAT). JAMA 2002; 288: 2981-2997.

19. Mabuchi H, Kita T, Matsuzaki M, Matsuzawa Y, Nakaya N, Oikawa S, et al; J-LIT Study Group. Japan Lipid Intervention Trial. Large scale cohort study of the relationship between serum cholesterol concentration and coronary events with low-dose simvastatin therapy in Japanese patients with hypercholesterolemia and coronary heart disease: Secondary prevention cohort study of the Japan Lipid Intervention Trial (J-LIT). Circ J 2002; 66: 1096-1100.

20. Berman DS, Kang X, Hayes SW, Friedman JD, Cohen I, Abidov A, et al. Adenosine myocardial perfusion single-photon emission computed tomography in women compared with men: Impact of diabetes mellitus on incremental prognostic value and effect on patient management. J Am Coll Cardiol 2003; 41: 1125-1133.

21. Haffner SM, Lehto S, Ronnemaa T, Pyorala K, Laakso M. Mortality from coronary heart disease in subjects with type 2 diabetes and in nondiabetic subjects with and without prior myocardial infarction. $N$ Engl J Med 1998; 339: 229-334.

22. Nishimura T, Nakajima K, Kusuoka H, Yamashina A, Nishimura S. Prognostic study of risk stratification among Japanese patients with ischemic heart disease using gated myocardial perfusion SPECT: J-ACCESS study. Eur J Nucl Med Mol Imaging 2008; 35: 319328.

23. Konishi M, Iso H, Iida M, Naito Y, Sato S, Komachi Y, et al. Trends for coronary heart disease and its risk factors in Japan: Epidemiologic and pathologic studies. Jpn Circ J 1990; 54: 428-435.

24. Rutan GH, Kuller LH, Neaton JD, Wentworth DN, McDonald RH, McFate Smith W. Mortality associated with diastolic hypertension and isolated systolic hypertension among men screened for the Multiple Risk Factor Intervention Trial. Circulation 1988; 77: 504-514.

25. Benetos A, Safar M, Rudnichi A, Smulyan H, Richard JL, Ducimetieère $\mathrm{P}$, et al. Pulse pressure: A predictor of long-term cardiovascular mortality in a French male population. Hypertension 1997; 30: 1410-1415.

26. Neaton JD, Wentworth D; for the Multiple Risk Factor Intervention Trial Research Group. Serum cholesterol, blood pressure, cigarette smoking, and death from coronary heart disease: Overall findings and differences by age for 316,099 white men. Arch Intern Med 1992; 152: 56-64.

27. Yasuno S, Ueshima K, Oba K, Fujimoto A, Hirata M, Ogihara T, et al. Is pulse pressure a predictor of new-onset diabetes in high-risk hypertensive patients? A subanalysis of the candesartan antihypertensive survival evaluation in Japan (CASE-J) trial. Diabetes Care 2010; 33: $1122-1127$.

28. The GISEN Group (Gruppo Italiano di Studi Epidemiologici in Nefrologia). Randomised placebo-controlled trial of effect of ramipril on decline in glomerular filtration rate and risk of terminal renal failure in proteinuric, non-diabetic nephropathy. Lancet 1997; 349: $1857-1863$.

29. Go AS, Chertow GM, Fan D, McCulloch CE, Hsu CY. Chronic kidney disease and the risks of death, cardiovascular events, and hospitalization. N Engl J Med 2004; 351: 1296-1305.

30. Ninomiya T, Kiyohara Y, Kubo M, Tanizaki Y, Doi Y, Okubo K, et al. Chronic kidney disease and cardiovascular disease in a general Japanese population: The Hisayama Study. Kidney Int 2005; 68: $228-236$.

31. Irie F, Iso H, Sairenchi T, Fukasawa N, Yamagishi K, Ikehara S, et al. The relationships of proteinuria, serum creatinine, glomerular filtration rate with cardiovascular disease mortality in Japanese general population. Kidney Int 2006; 69: 1264-1271.

32. Levey AS, Coresh J, Balk E, Kausz AT, Levin A, Steffes MW, et al; National Kidney Foundation. National Kidney Foundation practice guidelines for chronic kidney disease: Evaluation, classification, and stratification. Ann Intern Med 2003; 139: 137-147.

33. Ogihara T, Saruta T, Rakugi H, Fujimoto A, Ueshima K, Yasuno S, et al. Relationship between the achieved blood pressure and the incidence of cardiovascular events in Japanese hypertensive patients with complications: A sub-analysis of the CASE-J trial. Hypertens Res 2009; 32: 248-254.

34. Ueshima K, Oba K, Yasuno S, Fujimoto A, Sato Y, Fukiyama K, et al. Long-term effects of candesartan and amlodipine on cardiovascular mortality and morbidity in Japanese high-risk hypertensive patients: Rationale, design, and characteristics of Candesartan Antihypertensive Survival Evaluation in Japan Extension (CASE-J Ex). Contemp Clin Trials 2009; 30: 97-101. 